\title{
Interactive comment on "Using airborne HIAPER Pole-to-Pole Observations (HIPPO) to evaluate model and remote sensing estimates of atmospheric carbon dioxide" by C. Frankenberg et al.
}

\section{Anonymous Referee \#2}

Received and published: 9 March 2016

The paper "Using airborne HIAPER Pole-to-Pole Observations (HIPPO) to evaluate model and remote sensing estimates of atmospheric carbon dioxide" by Frankenberg et al. describes the comparison of HIPPO aircraft CO2 data with the CarbonTracker and MACC models and with the satellite instruments GOSAT, TES, and AIRS. Both total column dry air mole fractions and partial columns are considered. The emphasise of the study is to investigate how well models and satellite instruments perform in capturing background levels of methane in remote areas far away from local sources. The HIPPO campaigns are well-suited for these comparisons as the flights are largely over the oceans. 
The paper is concisely written and provides new results and insights in the performances of various models and instruments. The topic and presentation is well suited for ACP and I would recommend publication.

I have only identified some minor issues, but there is one point the authors should address somewhat more extensive; the mathematical treatment of the comparison, ie. the application of the averaging kernels and the extensions of the HIPPO profiles above flight altitude (see below for details).

Minor revisions:

p5, 18-10: This enables ... denoted XCO2 I think this statement would be more clear to the reader when a line is added to indicate that an extension is needed above $14 \mathrm{~km}$. Then you can indeed state that this extension is of limited consequence since most of the variability in XCO2 stems from the troposphere which is covered by the HIPPO profiles.

p7, I11-12: As most ... analysis. Add a line why SCIAMACHY does not provide data over oceans

p7, I16: short-wave $\rightarrow$ short-wave infrared

p8, 111: How can averaging lead to the reduction of systematic errors?

p9, 19-11: Validation ... (Olsen and Licata, 2014). If Olsen and Licata already have compared IR/MW L2 and IR-Only L2 against HIPPO, then I would expect a sentence explaining how the current study differs and/or extends wrt. the cited paper.

p9, 114-18: For the differences ... should dominate If you first extend the HIPPO profile with model data, then integrate, and finally subtract the integrated model data, does the part above flight altitude not exactly cancel? 
HIPPO $(<14 \mathrm{~km})$ - model $(<14 \mathrm{~km})$.

So, I do not see how the extension can contribute to the difference between HIPPO and model.

p10, 114-23: Figure 4 ... potential biases. HIPPO 3 is nicely explained in this paragraph, but HIPPO 5 is depicted in the Figure but not mentioned. Any comment that the authors can make on the MACC and CT differences/similarities?

p11, 12-10: Here, we look ... in the future. This alinea is mostly about measurements and campaigns that are not treated in the paper. I understand why the authors like to mention this, but maybe the conclusion, which includes a future outlook mentioning OCO-2, is the better spot for this.

p11, 111-19: For the comparison ... were the truth). This my strongest comment on the paper: Since the requirements on XCO2 are so stringent, it matters for the comparisons in this paper how exactly 1) the HIPPO profiles are extended, 2) the averaging kernel is applied, and 3) the null-space is attributed. I would recommend to incorporate a small section/paragraph explaining the mathematical details.

Questions that come to mind: Is the model information just attached to the HIPPO profile? If a jump would appear in such a profile, how is that treated? Is the smoothed (extended) HIPPO profile compared to the GOSAT profile without null-space contribution, or is there also a null-space contribution to the smoothed HIPPO profile? If the latter, which reference is used? The same as in the GOSAT retrievals, or the model?

p11, I22-24: Even after ... for MACC. Please refer to Figs 5 and 6

p11, I22: Even after normalization It is clear how the HIPPO data is corrected, but how is the other data corrected? With the HIPPO value, or with the average value of the particular model?

p13, I23: lower left quadrant Maybe the authors would like to note that these points are also outliers in the CT comparison. Not as strong as in the case of MACC, but still in 
the same quadrant, which may be an indication that the transport errors in both models are roughly equal and/or the GFED data is somewhat off.

p24, Fig 3: There are some strong excursions in the HIPPO profiles close to the surface; any explanation for these? For HIPPO-1, 3, and 4 (and possibly 5), the differences between HIPPO and MACC resp. CT differs significantly for $>70 \mathrm{~N}$. Any explanation for this behaviour? Please, reposition the legend box; CT-HIPPO 5 is barely visible.

Interactive

comment

p26-p28, Fig 5-7: Mention the shift for both axes

p31,p32, Fig 10,11: Mention the shift for both axes

Technical corrections:

p4, 15: Greenhouse Gas Observing $\rightarrow$ Greenhouse Gases Observing

p4, 15: haven $\rightarrow$ have

p4, I11: sensing measurement $\rightarrow$ sensing measurements

p5, 15-8: This sentence does not have a verb. Suggestion: The HIAPER Polo-to-Pole Observations (HIPPO) project consists of a sequence of ...

p6, I23: LSCE. To be on the safe side I would explicitedly write out this acronym

p9, 121-22: consistent between model, $\rightarrow>$ consistent between the two models,

p10, 16: usually $162253 \rightarrow$ usually

There are several places where ppb is used in stead of ppm: p11, 124 p11, I25 p21, Table 1 (2 instances)

p16, I11: that $\rightarrow$ than

Interactive comment on Atmos. Chem. Phys. Discuss., doi:10.5194/acp-2015-961, 2016. 